\title{
Training Simulation Models and Architectures in Power System Operation and Control
}

\author{
Róbert Gaál ${ }^{1}$, Attila Kovács ${ }^{1}$
}

\begin{abstract}
This paper summarizes - particularly the Hungarian experiences of dispatcher training simulation in the area of electrical power systems. The paper analyzes the widely different requirements of operators at different hierarchical levels of the network and suggests ways in which these might be met. Training simulation in electrical networks has to combine the flexibility of study simulation and the experience of events in real-time. The degree of detail and points of emphasis of the models can vary to a great extent. This depends on the role people trained on the simulator play in system supervision. The algorithms and methods outlined in this paper can fulfill these various requirements, and provide the possibility of modelling distribution or transmission networks, power plant household networks or railway traction power networks. The models can easily be extended with the feature of the simulation of renewable generation or micro grids as well.
\end{abstract}

\section{Keywords}

training simulation, network modelling, simulator architectures

\section{Acknowledgement}

The projects referred to in this article are supported by MAVIR Hungarian Independent Transmission Operator Company, E.ON Hungary, Paks Nuclear Power Plant Ltd. and Budapest Electricity Plc.

\section{Introduction}

Simulators are applied in several areas of power systems. Regarding the aim of the application these may be training- or study simulators.

- In the case of study simulators, the focus is on the accuracy and modelling details for research purposes, the way of visualisation of the results is less important. These kind of applications are not time critical ones. A complex computation may run for minutes or hours. According to the main domain of the research, even timeconsuming algorithms can be applied.

- The training simulator is a tool to improve the skills of a human operator. The main focus here is on the "real-world" experience of the user. Close or identical user interface to the real one is inevitable. Training simulation must happen in real-time or at least within the sense perception limits of the trainee. As a result, there are strict requirements especially concerning the simulation of electrical networks, where information propagates with light speed on the lines, and disturbances take place in a fraction of a second. Simulation - even on very powerful hardware - must spare with computing resources and only the most important details should be modelled. These models can also be less accurate. If a network is made of an extreme large number of components (e.g. up to 150.000 primary devices in a distribution network), realistic simulation can be reached only by consistent application of the principles of decomposition (re-calculate only the network segments where changes took place) and eventdriven computation (re-calculate only upon changes).

\footnotetext{
Astron Informatics Ltd.

H-1117 Budapest, Budafoki út 56, Hungary

(e-mail: gaal@astron.hu, kovacsa@astron.hu)
}

This is an Open Access article distributed in accordance with the Creative Commons Attribution Non Commercial (CC-BY-NC-ND 4.0) license, which permits others to copy or share the article, provided original work is properly cited and that this is not done for commercial purposes. Users may not remix, transform, or build upon the material and may not distribute the modified material (http://creativecommons.org/licenses/by-nc/4.0/) 
R. Gaál1, A. Kovács
Training Simulation Models and Architectures in Power System Operation and Control

\section{Application areas of training simulation}

\subsection{System operation}

The role of system operators is to monitor the transmission network, large generation units, the balance of generation and consumption (system frequency) and the international transaction schedules. Their scope of supervision is quite large, focused on system- and intersystem level processes, thus it's difficult for them to pay attention to all the details of the low level operation of primary (e.g. switching equipment) and secondary devices (protections, automations).

There is often a dedicated switching personnel (switching or network dispatchers) supporting the work of system operators, supervising the local technology of the bulk substations and transmission network, planning and executing switching sequences in order to reach a desired network topology in normal and emergency situations. Their activity should be closely co-ordinated with the system operator interactions.

Nowadays, according to the regulations of the European Union, it's obligatory to use simulators to facilitate the training of dispatchers.

Hungarian TSO system operators have trainings at least twice a year on Dispatcher Training Simulator (DTS). Its models put the emphasis on simulating system level phenomena as:

- Effective power - frequency model to describe frequency deviations due to the lack or surplus of generated effective power.

- Load-flow calculation according to the actual node infeedings and consumption.

- Automatic Generation Control model ensuring the balance of generation and consumption in the neighbouring control areas as well.

However, the DTS modelling described above is insufficient for the training of switching dispatchers, because of the lack of detailed simulation of primary and secondary devices. At the Hungarian TSO, the switching personnel is trained on a separate Network Training Simulator (NTS), which comprehensively simulates switching equipment (asymmetric switching states, drive errors, breaker stuck etc.) and event sequences due to protection and automation operation, with $1 \mathrm{~ms}$ resolution.

Establishing data exchange and synchronisation between DTS and NTS, it's possible to execute cooperative training sessions with the participation of both system- and switching dispatchers (Joint Simulator System [1], see Figure 1).

\subsection{Distribution network}

The main task of distribution network operators is to maintain the continuous supply of energy at the consuming points, quantified by the indices System Average Interruption Frequency Index (SAIFI) and System Average Interruption Duration Index (SAIDI).

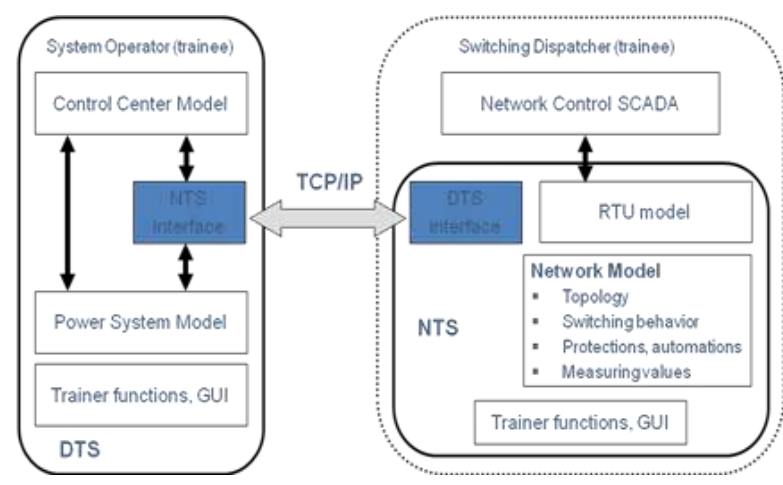

Figure 1

Their most important activities during disturbances are:

- Localisation of faults in a highly extended network.

- Executing the restoration via remote controls.

- Coordination of the staff involved in restoration (onsite personnel, fire-fighters etc.).

Distribution operators' interactions have direct effect on consumers, while system level processes (e.g. frequency balance) usually are not influenced by them. Thus, training simulators for distribution operators must focus to the next modelling points:

- Realistic simulation of the protection event sequences, and switching state changes, as these are what operators base their decisions on.

- Simulation of movement and activity of on-site maintenance team. Its communication role must be played by the trainer during the exercises.

- Modelling of measured values (i.e. medium voltage network calculation) may provide important supplementary information, but it is less relevant in distribution level training.

Instead of describing power system level phenomenon, a detailed local modelling is needed. For this reason, the NTS functionality described in 2.1 may be applied for simulating distribution networks as well [2]. However, medium voltage special features must be developed, and the hundred time greater number of primary and secondary devices may be a real challenge to reach the desired response time in every operational situation. 


\subsection{Railway traction power network}

In contrast with the training of railway traction power network operators, railway traffic control dispatchers are trained with simulators all over the world. However, outage of a traction power overhead line may cause severe interruption of traffic as well. That is why electric dispatchers should localize the error and restore the faulty line within a very limited period of time. Traction Power Network Training Simulator (TPNTS) is an appropriate tool to practise it.

The electrical network, primary and secondary equipment to be simulated here is very similar to the one of distribution technology, only a few special railway protections and automations must be replaced during modelling [3]. Also, instead of a three-phase network model, a single-phase one must be built. The timedependency of load (due to the moving of electric locomotives) is relevant and it can be simulated according to railway timetables.

Establishing data connection between TPNTS and the Traffic Control Dispatcher Training Simulator, cooperative training sessions can be organized for electrical and traffic control dispatchers.

\subsection{Power plant electrical simulation}

In power plants, the electric systems constitute only ten percent of the complete technology, but their availability is crucial. Without electricity supply, mechanical auxiliary systems will be outaged, too. That is very critical especially in nuclear power plants, where existence of cooling water and the availability of pumping system are the two top-priority safety issues. Anyway, nuclear power plants may not be started up without outer auxiliary electricity sources at all (black-start).

Thus, besides the simulation training of main technological systems (e.g. nuclear reactor, primary and secondary water-/steam circuits), developing the skills of electrical operators is also inevitable. The Power Plant Network Training Simulator (PPNTS) beyond main electrical units (generator, excitation system, block transformer) has to simulate the household distribution network, too. As an example, the PPNTS of nuclear power plant Paks, Hungary simulates 16 generators of the 8 generation units, the $6 \mathrm{kV}$ and $0,4 \mathrm{kV}$ household network behind with main and spare feedings and the diesel generator backup system [4]. Electrical operators may be prepared for handling various normal and disturbance operational states, including:

- $\quad$ tripping of a single generation unit,

- complete loss of voltage,
- black-start of the plant from outer voltage source.

Here, synchronizing generators to the adjacent network is only a final action of a long restoration sequence. Modelling requirements - excepting the simulation of main electrical units (generator, excitation) - are similar to the ones of distribution networks (see 2.2), so enhanced NTS simulation models can be applied.

\subsection{Simulation of micro-grids}

In the close future, by spreading the renewable distributed energy generation and storage technology, micro-grids will become more and more relevant components of power systems. In the analysis of their operation and interaction with the adjacent network, the application of simulation is indispensable.

A micro-grid can be considered as a mini power system, having its own effective power - frequency and voltage control, but it can be decoupled from the network only in very special cases. However, using its generating capacities and intelligent consumers, it is able to follow transaction schedules.

When simulating micro-grids - besides describing (medium or low voltage) distribution network components (lines, cables, switching equipment) - a special emphasis must be put on modelling:

- Renewable generation units (solar cells, wind- and water turbines) including the simulation of weather conditions.

- Consuming units; due to their small amount, loads must be modelled not statistically but uniquely (e.g. a heat pump of a large building).

- Storage unit (large-capacity accumulator), that can both generate or consume energy.

- Micro-grid control system monitoring inner generating-, storage- and consuming units (it can optimize the energy purchase from the adjacent network, set up islanding operational state etc.).

As a good example, Liveable Future Park Fót, Hungary [5], sponsored by the local electricity supplier, has a low-voltage micro-grid connected to the medium voltage utility network, comprising renewable generating units, consumers and a SCADA system. As part of the project, a Smart Network Training Simulator was realized (SNTS), allowing researchers to study various controlling schemas in different weather conditions, without disturbing everyday life of the Park.

An attractive simulation user interface was developed as well, to demonstrate micro-grid operation for the visitors. 


\section{Simulation models, modelling principles}

When designing simulator models for different applications detailed in Chapter 2, the following contradictory aspects must be taken into account:

- Accuracy and details of the models.

- Physical extent of the models (network size, simulated voltage levels, number of elements).

- Desired response times.

These points can be summarized as the scope of modelling. Further considerations are costs (hardware, software, development) and the duration of the implementation.

For training simulation, the adequate scope of modelling must always be derived from the tasks of dispatchers in normal and emergency conditions. If we move down in power control hierarchy, its focus area changes from system level processes to the operation details of single devices (switchgears, protections etc.), as it was presented in Chapter 2.

Training simulation modelling for electrical networks must cover the next main domains:

- Stationary and transient state modelling of the network (i.e. simulating continuous changing of analogous values, as current, voltage, power, frequency).

- Time-discrete modelling the operation of primary and secondary network devices.

\subsection{Continuous modelling of analogous values}

Electrical transients appearing in the network due to switching actions or short-circuits are not relevant from a modelling point of view, as these take place so quickly, that neither dispatchers nor data acquisition systems (RTUs, SCADA) are able to sense it. Network state variables (current, voltage, power, frequency) can be modelled with the sequence of stationary states before and after changes (quasi-stationary model). Network state variables in radial networks can be calculated with the method of nodal potentials and in meshed networks with different iterative algorithms (Gauss-Seidel, NewtonRaphson).

Inside the substations, the load-flow algorithms cannot be applied, as there may be several branches with nearly zero impedance (but their current is measured in reality, so we have to simulate it). Here nodal potential method is appropriate, however some heuristic approach is allowed. The results of the load-flow of the adjacent network must be used as input values of the substation's inner calculation, thus keeping our model fully coherent.

\subsection{Discrete modelling of network devices}

For detailed modelling of single network devices (circuit breakers, isolators, protections, automations, interlocks etc.) the method of Synchronous Sequential Networks (SSNs) can be applied, as the outputs of these devices depends only on their inputs and their previous states (the operation of a line auto-recloser is a good example). In this approach, a combination formula represents the behaviour of a device describing the relations between inputs, outputs and state variables. The models of single devices can be connected to each other as the real ones (see Figure 2). (Device outputs can be fed back to its own inputs, as well.) An output of a device (e.g. a protection tripping signal) may change the input of another one (e.g. a circuit breaker switching OFF). In this case the combination formula representing the device of the changed input must be recalculated to generate its new outputs (breaker OUT state signal). This sequence should be repeated until reaching a steady state.

A dedicated algorithm (Discrete Simulation Engine, DSE) was developed to cyclically evaluate the combination formulas of devices and propagate changes according to the configuration of SSN. Here, discrete simulation cycle corresponds to the clock signal of the synchronous network.

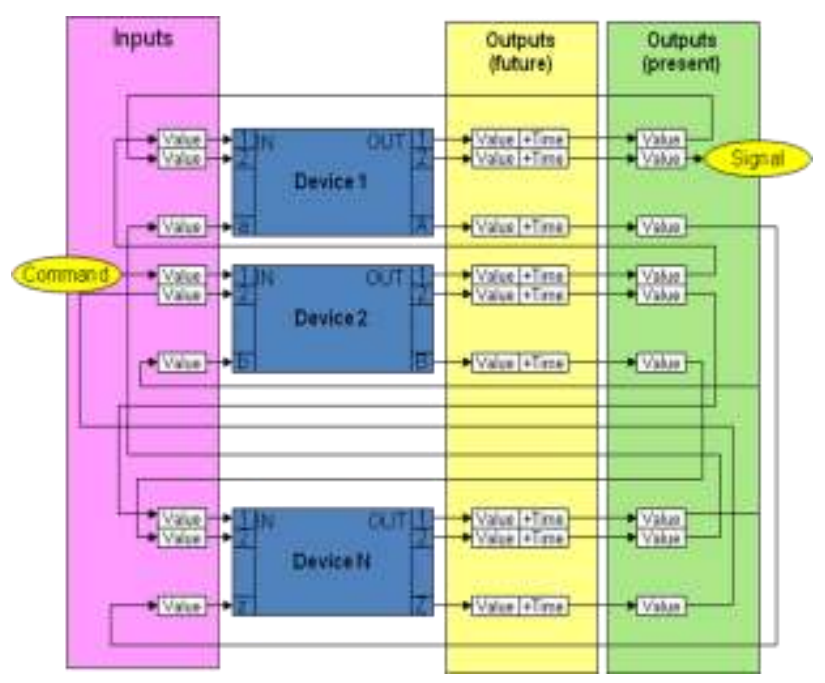

Figure 2

DSE is also responsible for managing simulation time and generating time-stamps of the different signals and alarms (protection trip, breaker out, auto reclosing etc.) with millisecond resolution.

\subsection{Modelling topology}

The following two-level topology description establishes a relationship between continuous (i.e. quasistationary) and discrete models: 
The detailed topology model is composed of all the network components and its connections, including busbars, lines, transformers, measuring transformers, switching devices etc. Every single primary component of the inside of substations (busbar sections, wire sections in feeders etc.) must be separately described. Discrete models according to 3.2 and node potential method calculating the substation's inner flows and voltages (see 3.1) use detailed topology model.

The bus-branch model can be derived from the detailed topology model with merging substation components into one or a few network nodes and branches of large impedance between them (transmission lines, transformers). Network calculation algorithms (see 3.1) use this bus-branch model.

During topology changes the models above should be updated to- and backward, dynamically. For example models of protections and automations detect shortcircuits at measuring points (measuring transformers) and change switching device statuses, which modifies the busbranch level topology as well. Thus network calculation will be run and its results will be stored in measuring transformer elements of the detailed topology model.

In reality, protections localize and classify shortcircuits via the analysis of transient current- and voltage curves. This functionality must not be implemented, as in the simulator the topological location of short-circuits is well-known, since these were generated by a trainer (or a scenario). For this reason, the protection system model may simulate short-circuit detection in a logical way and not via measuring physical values.

The detailed topology model must support topology analysis in order to predict the outcome of switching operations that can be a new normal operational state or a switching fault (ground short-circuit, load disconnecting with isolator). For detecting switching faults, a set of topology analyzing algorithms described in [6] were applied in the applications of Chapter 2. This way of simulating switching behaviour of the network may be considered as an algorithmic extension of topology modelling. In practice, the interlocking system is responsible for preventing switching faults, but in the simulation environment, the faults of interlocking can be modelled as well, or interlocks can be switched off completely for didactical purposes.

\section{Simulator architectures}

On the left side of Figure 3, the main functional modules of a training simulator can be seen. On the right side, the mapping of them to hardware components is shown. For the sake of realistic trainee GUIs, it is very common to use a copy of the existing SCADA system instead of re-implementing a Control Center Model (CCM). Thus CCM is realised not via simulation but emulation. For cost efficiency reasons, all other model parts are simulated (not emulated) in a separate hardware. The simulator server is connected to the trainee SCADA via standard data acquisitioning interface, usually with the networked IEC 850-7-104 protocol, providing the possibility of simulation of a large number of RTUs at a time.

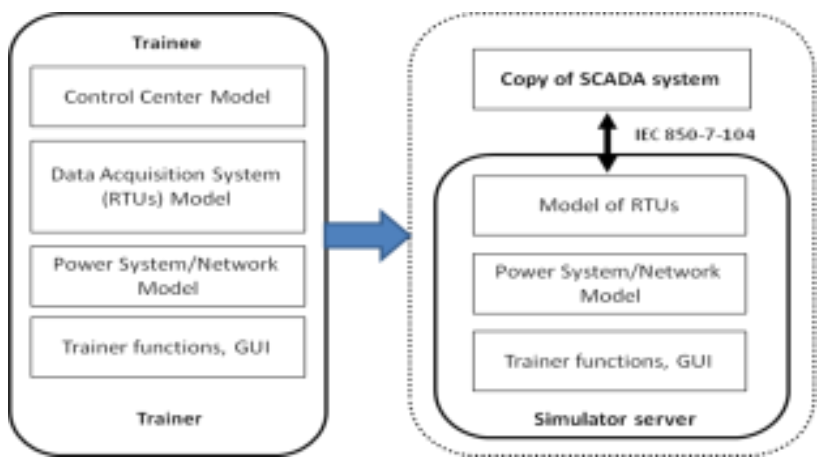

Figure 3

The advantages of this structure are:

- If we simulate a real network or a part of it, trainee SCADA engineering efforts will not arise.

- It is easy to set up an integrated architecture, where the simulator server is connected to the front-end system of a live SCADA system.

- Due to the standard interface, the simulator can be connected to any type of SCADA systems.

- The simulator server works without the trainee SCADA as well, so for demonstration purposes it can be installed on a portable computer.

\section{Conclusion}

Dispatcher training supported by simulators is inevitable even in the lower levels of power system control hierarchy. However, in the case of different applications, modelling requirements change based on the various roles of dispatchers. Simulation models must be designed and realised according to the fact, that the focus of operator activities below system level is shifted to the operational details of primary and secondary devices. 
R. Gaál1, A. Kovács

\section{References}

[1] Sztráda, Gy., Kovács, A., "Joint Simulator System of Hungarian TSO MAVIR and its Application to the Training of Dispatchers", Elektrotechnika, 2011/09, pp. 11-13.

[2] Patócs, T., "Network Training Simulator of E.ON", $62^{\text {nd }}$ Annual Conference of Hungarian Electrotechnical Association, Siófok, Hungary, September 16-18, 2015.

[3] Gaál, R., Kovács A., "Traction Power Network Training Simulator in Dispatcher Training", InnoRail 2013 Budapest International Conference, Budapest, Hungary, October 28-30, 2013, poster paper.
[4] Gaál, R., Lakatos, G., "Co-operative Training for Black-start of Nuclear Power Plant Paks", 59th Annual Conference of Hungarian Electrotechnical Association, Budapest, Hungary, September 5-7, 2012.

[5] Gaál, R., "Simulation Modeling of Renewable Energy Park Low-voltage Network", 60nd Annual Conference of Hungarian Electrotechnical Association, Mátraháza, Hungary, September 11-13, 2013.

[6] Kovács, A., "Simulation of substations based on nonnumerical methods", International Journal of Engineering Intelligent Systems, December 1993, pp. 167-171. 